\title{
Interference of cefoxitin in the creatinine estimation and its clinical relevance
}

\author{
S. R. DURHAM ${ }^{1}$, A. H. C. BIGNELL, AND R. WISE \\ From the Departments of Medical Microbiology and Clinical Chemistry, Dudley Road Hospital, \\ Birmingham B18 7QH, UK
}

SUMMARY Serum creatinine and creatinine clearance were measured in 10 healthy volunteers before and after an intravenous injection of $2 \mathrm{~g}$ cefoxitin sodium. Results were compared with in vitro work which demonstrated a positive interference by cefoxitin in the Jaffé reaction, the routine laboratory method of creatinine measurement. The serum creatinine estimation should be delayed until at least 2 hours after cefoxitin administration. The creatinine clearance may appear to be falsely high.

Several of the cephalosporin antibiotics have been shown to react in the same manner as creatinine in the Jaffé test, the commonly used laboratory method for estimating creatinine in serum and urine (Swain and Briggs, 1977). Such antibiotics include cephaloglycin, cephalothin, cephaloridine, and cephacetrile, but the effect is most marked with the new cephamycin, cefoxitin (Mefoxin). The Jaffé reaction (DiGiorgio, 1974) depends on the interaction of creatinine and an alkaline solution of sodium picrate to produce a red-coloured compound, which is measured photometrically. The method is nonspecific as many substances present in normal human serum and urine may contribute to the colour formation (Butler, 1975). The nature of the colour complex formed is unknown, and hence the mechanism of interference by cephalosporins and other substances is not understood.

Studies in animals (Lawson et al., 1972; Linton et al., 1972) and in man (Foord, 1970; Carling et al., 1975) have shown certain of the cephalosporins, notably cephaloridine, to be nephrotoxic. This effect is usually observed when high-dosage regimes are used and is potentiated by the presence of previous renal impairment and the use of potent diuretics. Evidence of the potentiation of aminoglycosideinduced nephrotoxicity when cephalosporins are used in combination is controversial (Gaya et al.,

${ }^{1}$ Present address: Department of Cardiovascular Medicine, Hammersmith Hospital, Ducane Road, London, UK

Received for publication 10 April 1979
1975; Giamarellou et al., 1977). Cefoxitin, however, has been shown to be free of nephrotoxicity when used in moderate doses and in patients with mildly impaired renal function (Fillastre et al., 1978 Trollfors et al., 1978).

When cephalosporins are used it may be importane to monitor closely renal function. Serum creatinine and creatinine clearance are commonly used clinical tests. The in vitro studies of Swain and Briggs (1977) suggest that creatinine estimations might give a false impression of renal function when cefoxitin is being administered. This study was designed to investigate this possibility by a combined in vitro and in vivo study using human volunteers.

\section{Method}

Sera from a number of healthy individuals were pooled, and the creatinine value was estimated. The creatinine levels were re-estimated after spiking aliquots of this serum with cefoxitin to final concentrations of $100,250,500$, and $1000 \mathrm{mg} / 1$. Similarly pooled human urine had creatinine estimations performed unspiked and spiked with 1000, 2500, 5000 , and $10000 \mathrm{mg} / \mathrm{l}$ of cefoxitin.

Ten volunteers, six men and four women aged 21 to 35 years, mean weight $67 \mathrm{~kg}$, were studied over two consecutive days. The volunteers had normal full blood counts, biochemical profiles, and creatinine levels. They had no history of allergy or renal disease and were not taking concurrent medication (other than the contraceptive pill).

On day 1 (control day), after complete voiding 
of urine, an 8-hour urine collection was started. At 2 hours blood was drawn from an antecubital vein. Both blood and urine samples were sent for creatinine estimation and the creatinine clearance was calculated.

On day 2, $2 \mathrm{~g}$ of cefoxitin sodium (Merck Sharp and Dohme, Hoddesdon, UK) in $10 \mathrm{ml}$ sterile water was given to each volunteer over 2-3 minutes into an antecubital vein. No pain was experienced at the injection site. Blood was drawn from the same needle into another syringe immediately after drug administration (time $\mathbf{0}$ minutes) and thereafter from the opposite antecubital fossa at 5 minutes, 2 hours and 4 hours. An 8-hour urine collection (starting after cefoxitin had been administered) was taken as for day 1. All samples were sent for creatinine and also cefoxitin estimation.

Throughout the study volunteers were on free diets, avoided excessive physical exertion, and were encouraged to drink approximately 1.5 litres of fluid over the 8 hours in order to assist standardisation of the urine volume collected.

All serum and urine creatinine estimations were by the Jaffé reaction by the standard AutoAnalyzer I procedure (Technicon Methodology N116), which incorporates a dialysis step.

The standard plate diffusion technique, using Bacillus subtilis as indicator organism and 'Oxoid' antibiotic No. 1 media, pH $7 \cdot 2$, was used for the cefoxitin estimations after making appropriate dilutions of the serum and urine samples.

\section{Results and discussion}

The effect on the creatinine level of adding cefoxitin to normal serum and urine is shown in the Figure. From this the mean creatinine equivalence of 1000 $\mathrm{mg} / \mathrm{l}$ of cefoxitin was found to be $152 \mu \mathrm{mol} / \mathrm{l}$. In urine, the creatinine equivalence of $1000 \mathrm{mg} / \mathrm{l}$ of cefoxitin was $155 \mu \mathrm{mol} / \mathrm{l}$.

Values for serum creatinine are shown in Table 1. Student's paired $t$ test was used to compare the values on day 2 with those on day 1 of the study. A highly significant increase was noted at 0 and 5 minutes but at 2 and 4 hours the differences were not significant. Urine volumes passed over 8 hours on day 1 (mean $1281 \mathrm{ml} \pm$ SD $473 \mathrm{ml}$ ) and day 2 $(1047 \mathrm{ml} \pm \mathrm{SD} 463 \mathrm{ml})$ showed no significant

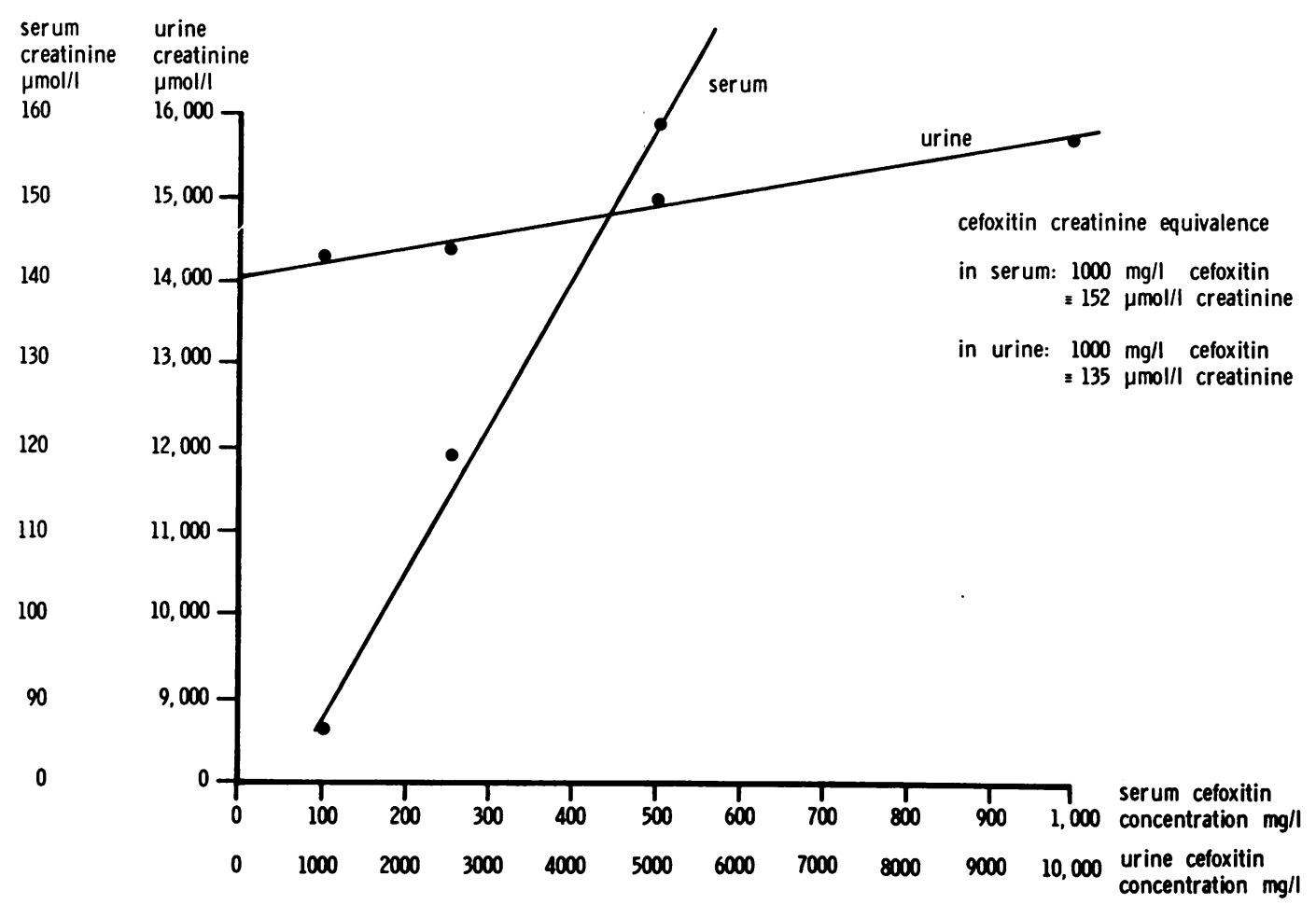

Figure Effect of differing concentrations of cefoxitin upon the apparent serum creatinine or apparent urine creatinine estimation. 
Table 1 Serum creatinine values for 10 volunteers ( $\mu$ molll) on day 1 (control) and day 2 (at times shown after $2 \mathrm{~g}$ cefoxitin intravenously)

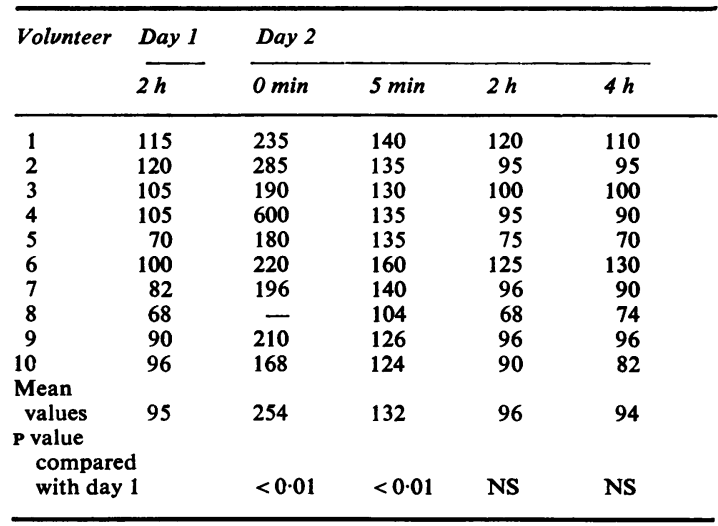

NS $=$ not significant

difference. Of the administered dose of cefoxitin $70.5 \%$ was excreted in the urine over the 8-hour period. The mean total urine creatinine output over 8 hours for day 1 was $3800 \mu \mathrm{mol}$ (SD 1003), and the apparent mean total output for day 2 was 7028 $\mu \mathrm{mol}$ (SD 2182), which is a highly significant increase $(P<0.01)$. Creatinine clearances and cefoxitin levels were determined, and mean values are shown in Table 2. The creatinine clearances calculated using the 2 and 4 hour serum samples were significantly different from the control day value.

Table 2 Means and standard deviations of creatinine clearance values ( $\mathrm{ml} / \mathrm{min}$ ) for 10 volunteers using ser um creatinine estimations at times shown and the serum cefoxitin levels

\begin{tabular}{|c|c|c|c|c|c|}
\hline & \multirow{2}{*}{$\frac{\text { Day } 1}{2 h}$} & \multicolumn{4}{|l|}{ Day 2} \\
\hline & & $0 \mathrm{~min}$ & $5 \min$ & $2 h$ & $4 h$ \\
\hline \multirow{5}{*}{$\begin{array}{l}\text { Mean creatinine } \\
\text { clearance } \\
\text { SD } \\
\text { P value compared } \\
\text { with day } 1 \\
\text { Mean serum } \\
\text { cefoxitin levels } \\
\text { day } 2(\mu \mathrm{g} / \mathrm{ml}) \\
\text { SD }\end{array}$} & 85 & 65 & 111 & 152 & 154 \\
\hline & 21 & 21 & 33 & 41 & 42 \\
\hline & & NS & NS & $<0.01$ & $<0.01$ \\
\hline & & 934 & 188 & $7 \cdot 5$ & 1.5 \\
\hline & & 693 & 44 & $2 \cdot 2$ & 0.5 \\
\hline
\end{tabular}

NS $=$ not significant

If the in vivo serum creatinine equivalence of cefoxitin is calculated from the difference in mean creatinine levels for day 1 at 2 hours and those on day 2 at 0 and 5 minutes, values of 170 and 196 $\mu \mathrm{mol}$ respectively per gram of cefoxitin are obtained. These values compare favourably with the in vitro value of $204 \mu \mathrm{mol} / \mathrm{g}$ of cefoxitin found by Swain and Briggs (1977) and close to the result of our own in vitro estimation quoted above. However, equating the mean increase in total apparent urine creatinine output $(3228 \mu \mathrm{mol})$ to the mean urine cefoxitin level $(1336 \mu \mathrm{mol})$ results in a creatinine equivalence of $2416 \mu \mathrm{mol} / \mathrm{g}$ of cefoxitin, that is, of the order of 10 times greater than the serum or urine cefoxitincreatinine equivalent.

Serum creatinine has been shown to be remarkably constant for the same individual (Haugen and Blegen, 1953; 1955; Sirota et al., 1950) within the limits $\pm 10 \mu \mathrm{mol} / 1$ and only slightly affected by diet (Annino and Relman, 1959). Similarly, urinary creatinine output does not vary from day to day, is independent of the volume of diuresis, and only slightly increased by diet and exercise (Van Pilsum and Seljeskog, 1958). In our study, apart from diet which has only a minimal effect when a high-protein intake occurs, these potential influences were standardised. Consequently, any variation in the creatinine studies of day 1 and day 2 must be attributed to the influence of cefoxitin.

The mean serum creatinine at time 0 minutes simulates the situation of unknowingly withdrawing blood for creatinine at the time of drug administration, thereby increasing the apparent serum creatinine by $159 \mu \mathrm{mol} / 1$. At 5 minutes, which corresponds to peak serum cefoxitin levels (188 $\mu \mathrm{g} / \mathrm{ml}$ ), the serum creatinine is increased by 37 $\mu \mathrm{mol} / \mathrm{l}$. Since the half-life of cefoxitin is fairly short, 40-60 minutes (Schrogie et al., 1978), the levels have fallen by 2 hours so that no significant effect on the serum creatinine is found.

The urine total creatinine outputs were considerably increased, out of proportion to the observed in vitro creatinine-like reaction of cefoxitin. This effect is difficult to explain. An increase in endogenous creatinine production from muscle would be reflected in serum levels and is therefore unlikely. It is possible that cefoxitin increases renal tubular secretion of creatinine, or, alternatively, perhaps a microbiologically inactive metabolite of cefoxitin in urine exerts a greater creatinine-like effect. This latter explanation is unlikely as the antibiotic is said to be excreted unchanged (Schrogie et al., 1978). The tubular secretion of creatinine is very low and could not be expected to account for the observation. It is possible that as urine is being formed a chemically active compound is produced which further interferes with the in vivo measurement of creatinine. Whatever the mechanism, the consequences are clear, namely, a wide variation in creatinine clearance, depending on the timing of serum sample used in the estimation. Interestingly, creatinine clearance based on the use of serum 
samples at 0 and 5 minutes was within the normal range, this being due to the plasma and urine creatinine-reactivity of cefoxitin tending to cancel each other in the calculation of clearance.

Creatinine clearance studies were carried out over an 8-hour period using a single dose of cefoxitin. However, since the antibiotic is usually given throughout the 24-hour period at 8-hourly intervals, its effect on creatinine excretion would be expected to continue throughout the day. The 8-hour creatinine clearance is, therefore, likely to be representative of the more usual 24-hour clearance measurement.

In conclusion, cefoxitin in therapeutic doses exerts a profound effect on creatinine studies, as measured by the Jaffé method, in normal human volunteers. Creatinine clearance becomes unreliable as an index of renal function in patients on cefoxitin. Serum creatinine may still be used provided the sample for analysis is withdrawn longer than 2 and preferably 4 hours after administration of the drug; this might not apply in renal impairment where accumulation of the drug occurs. Creatinine clearance estimations should not be relied on clinically. By inference, other cephalosporins, which have rather less creatinine-like reactivity in vitro, might exert less, though still clinically significant effects. In this situation, we recommend that an alternative assessment of renal function should be employed.

\section{References}

Annino, J. S., and Relman, A. S. (1959). The effect of eating on some of the clinically important chemical constituents of the blood. American Journal of Clinical Pathology, 31, 155-159.

Butler, A. R. (1975). The Jaffé reaction. Identification of the coloured species. Clinica Chimica Acta, 59, 227-232.

Carling, P. C., Idelson, B. A., Casano, A. A., Alexander, E. A., and McCabe, W. R. (1975). Nephrotoxicity associated with cephalothin administration. Archives of Internal Medicine, 135, 797-801.

DiGiorgio, J. (1974). Non-protein nitrogenous constituents. In Clinical Chemistry: Principles and Technics, edited by R. J. Henry, D. C. Cannon, and J. W. Winkelman, 2nd edition, pp. 541-553. Harper and Row, Hagerstown, Maryland, USA.

Fillastre, J. P., Leroy, A., Godin, M., Oksenhendler, G., and Humbert, G. (1978). Pharmacokinetics of cefoxitin sodium in normal subjects and in uraemic patients. Journal of Antimicrobial Chemotherapy, 4, Supplement B, 79-83.
Foord, R. N. (1970). Cephaloridine and the kidney. In Progress in Antimicrobial and Anticancer Chemotherapy: Proceedings of the 6th International Congress of Chemotherapy, 1969, vol. 1, pp. 597-604. University of Tokyo Press, Tokyo.

Gaya, H., Klastersky, J., and Schimpf, S. (1975). Nephrotoxicity during combined chemotherapy with gentamicin and cephaloridine. 15th Interscience Conference on Antimicrobial Agents and Chemotherapy, Washington, DC. September 24-26, 1975. Abstract 401.

Giamarellou, H., Pefrikkos, G., Dandonlaki, P., and Daikos, G. K. (1977). Prospective evaluation of gentamicin toxicity in humans. 10th International Congress of Chemotherapy, Zurich, September 18-23, 1977. Abstract 271.

Haugen, H. N., and Blegen, E. M. (1953). The true endogenous creatinine clearance. Scandinavian Journal of Clinical and Laboratory Investigation, 5, 67-71.

Haugen, H. N., and Blegen, E. M. (1955). Plasma creatinine concentration and creatinine clearance in clinical work. Annals of Internal Medicine, 43, 731-739.

Lawson, D. H., Macadam, R. F., Singh, H., Gavras, H., Hartz, S., Turnbull, D. I., and Linton, A. L. (1972). Effect of furosemide on antibiotic-induced renal damage in rats. Journal of Infectious Diseases, 126, 593-600.

Linton, A. L., Bailey, R. R., and Turnbull, D. I. (1972). Relative nephrotoxicity of cephalosporin antibiotics in an animal model. Canadian Medical Association Journal, 107, 414-416.

Schrogie, J. J., Davies, R. O., Yeh, K. C., Rogers, D., Holmes, G. I., Skeggs, H., and Martin, C. M. (1978). Bioavailability and pharmacokinetics of cefoxitin sodium. Journal of Antimicrobial Chemotherapy, 4, Supplement B, 69-78.

Sirota, J. H., Baldwin, D. S., and Villarreal, H. (1950). Diurnal variation of renal function in man. Journal of Clinical Investigation, 29, 187-192.

Swain, R. R., and Briggs, S. L. (1977). Positive interference with the Jaffé reaction by cephalosporin antibiotics. Clinical Chemistry, 23, 1340-1342.

Trollfors, B., Norrby, R., and Kristianson, K. (1978). Effects on renal function of treatment with cefoxitin sodium alone or in combination with furosemide. Journal of Antimicrobial Chemotherapy, 4, Supplement B, 85-89.

Van Pilsum, J. F., and Seljeskog, E. L. (1958). Long term endogenous creatinine clearance in man. Proceedings of the Society of Experimental Biology and Medicine, 97, 270-272.

Requests for reprints to: Dr R. Wise, Department of Medical Microbiology, Dudley Road Hospital, Dudley Road, Birmingham B18 7QH, UK. 